DOI 10.37882/2500-3682.2021.01.13

\title{
ПСИХОЛОГИЧЕСКИЕ ОСОБЕННОСТИ ЛЮДЕЙ, ПРЕДРАСПОЛОЖЕННЫХ К ПАНИЧЕСКИМ АТАКАМ И ТРЕВОЖНЫМ РАССТРОЙСТВАМ
}

\section{PSYCHOLOGICAL CHARACTERISTICS OF PEOPLE PREDISPOSED TO PANIC ATTACKS AND ANXIETY DISORDERS}

\section{K. Semikova}

Summary: The article is devoted to the problem of panic attacks and anxiety disorders, compiled based on official original sources and publications in foreign media. The article discusses the relationship between the development of panic attacks and the level of autoaggression, aggressive and defensive position. Influence of disorders of emotion regulation on the development of panic attacks and stress tolerance. Features of the influence of birth injuries on the formation of panic attacks and aggression.

Keywords: panic, panic attacks, anxiety, distress tolerance, anxiety disorders, depression.

\author{
Семикова Ксения Владимировна \\ Старший преподаватель, Еврейский Университет, \\ 2. Москва \\ k.semikova@chamesh.ru
}

Аннотация: Статья посвящена проблеме панических атак и тревожных расстройств, составлена на основе официальных оригинальных источников и публикаций в зарубежных СМИ. В статье рассматривается взаимосвязь развития панических атак с уровнем аутоагрессии, агрессивно-защитной позицией. Влияние нарушений регуляции эмоций на проживание панических атак и стрессоустойчивости. Особенности влияния родовых травм на формирование панических атак и агрессии.

Ключевые слова: паника, панические атаки, тревожность, стрессоустойчивость, тревожное расстройство, депрессия.
$\mathrm{H}$ астоящая статья посвящена проблеме панических атак и тревожных расстройств, составлена на основе официальных оригинальных источников и публикаций в зарубежных СМИ и является частью исследовательского проекта автора на тему: «Психологические условия коррекции эмоциональной сферы людей, предрасположенных к паническим атакам».

Основу статьи составили публикации на сайтах ведущих мировых каналов и онлайн изданий, таких как Social Psychiatry and Psychiatric Epidemiology,BMC Psychiatry volume, International Journal of Cognitive Therapy, Cognitive Therapy and Research volume, Clinical Psychological Science - представляющие интерес для проводимого исследования.

При отборе материалов автор придерживался следующих принципов и критериев: соответствие тематике научной работы, новизна, практическая значимость

Актуальность темы обусловлена нарастающими нагрузками на нервную систему и психику современного человека, которые приводят к формированию эмоционального напряжения. Оно выступает одним из главных факторов развития различных психосоматических заболеваний. В настоящее время на первый план выходит забота о сохранении психического здоровья и формировании эффективных способов преодоления деструктив- ных эмоциональных состояний современными методами коррекции.

Нарастающие нагрузки на нервную систему и психику современного человека приводят к формированию эмоционального напряжения, которое выступает одним из главных факторов развития различных психосоматических заболеваний. В настоящее время на первый план выходит забота о сохранении психического здоровья и формирования эффективных способов преодоления деструктивных эмоциональных состояний современными методами коррекции.

Тревожно-фобические расстройства относятся к наиболее распространенным формам пограничной психической патологии; ими страдает до 6\% населения. В последнее время в медицине и психологии возрос интерес исследователей к данным состояниям, среди которых особое внимание привлекают панические расстройства, наиболее ярким и драматичным проявлением которых являются панические атаки. Этот интерес определяется негативным влиянием панических расстройств на качество жизни и социальную адаптацию, низкой эффективностью существующих методов диагностики и лечения этих состояний.

По данным, как российских, так и зарубежных ученых, панические расстройства встречаются у 1,5-3\% 


\section{взрослого населения.}

Изучение панических расстройств свидетельствует о том, что многие аспекты этой важной проблемы все еще далеки от своего разрешения. До настоящего времени нет единства в терминологическом обозначении панических расстройств, существует множество способов коррекции, неясны причины формирования данных расстройств.

Панические атаки или вегетативный криз - представляет собой рационально необъяснимый практически неконтролируемый, и, как бы, случайный приступ тревоги, сопровождаемый страхом вместе с различными соматическими проявлениями: онемение отдельных участков тела или всего тела, дрожь, заторможенность реакций и прочие симптомы. Любопытным является тот факт, что страх часто, но не всегда, является не предметным, то есть скорее речь идёт о переживании беспричинной тревоги крайне высокой интенсивности.

Отечественные исследователи традиционно квалифицируют панические расстройства как синдром, встречающийся при различных психических заболеваниях - шизофрении, маниакально-депрессивном психозе, неврозах, алкоголизме, а также в рамках синдрома соматоформной вегетативной дисфункции.

Важно отметить, что «паническая атака», практически никогда не является самостоятельным диагнозом, а является лишь одним из указателей на наличие какоголибо психологического, психического или физиологического расстройства и нередко входит в контекст того или иного психического расстройства и ряда сложных психологических состояний кризисного спектра.

Выделяют три основных разновидности панических атак:

1. Спонтанные панические атаки, возникающие неожиданно, без видимых причин;

2. Ситуационные панические атаки, спровоцированные переживаниями определенной психотравмирующей ситуации, либо же чувством её ожидания;

3. Условно-ситуационные панические атаки, возникающие чаще под действием биологического или химического стимула (прием алкоголя, перестройка гормонального фона при менструальном цикле).

В зарубежной литературе панические расстройства представлены на синдромальном уровне, в основном в связи с проблемой коморбидных состояний.

В данной работе панические атаки будут рассматриваться преимущественно с точки зрения психологии, как особый вид тревожного состояния (симиотомокомплекс на основе переживания тревоги).

В контексте проблематики исследования анализировались зарубежные исследования в области психологического консультирования, клинической психологии и психотерапии, исследования природы тревожности и сопутствующих феноменов. Срединих: Andrew J. Baillie, Ronald M.Rapee. «Panic attacks as risk markers for mental disorders», Arthur Janov. "THE ORIGIN S OF AN XIETY, PAN IC AND RAGE ATTACKS», Claire M Kelly 1, Anthony F Jorm, Betty A Kitchener. «Development of mental health first aid guidelines for panic attacks: a Delphi study», Gulay Oguz, Zekiye Celikbas,Sedat Batmaz, Saime Cagli1,Mehmet Zihni Sungur. "Comparison Between Obsessive Compulsive Disorder and Panic Disorder on Metacognitive Beliefs, Emotional Schemas, and Cognitive Flexibility», Kirsten A. Johnson, Erin C. Berenz, Michael J. Zvolensky. «Nonclinical Panic Attack History and Anxiety Sensitivity: Testing the Differential Moderating Role of Self-Report and Behavioral Indices of Distress Tolerance», Lynne Lieberman, Stephanie M Gorka , Stewart A Shankman, K Luan Phan . «Impact of Panic on Psychophysiological and Neural Reactivity to Unpredictable Threat in Depression and Anxiety», Matthew T. Tull. «Extending an Anxiety Sensitivity Model of Uncued Panic Attack Frequency and Symptom Severity: The Role of Emotion Dysregulation»

В настоящее время тема панических атак очень актуальна. Заболевание как правило начинается остро и быстро хронифицируется, вызывая значительную социальную дезадаптацию и снижение качества жизни. Панические атаки часто сочетается с другими формами психопатологии. У $30 \%$ больных паническими расстройствами отмечается депрессия, у $42 \%$ тревожные и соматоформные расстройства, в 34-58\% случаев ПА сочетаются с алекситимией, в 68\% - с агорафобией, которая значительно ограничивает социальную активность. Развивающееся ограничительное поведение препятствует профессиональной деятельности.

В результате анализа исследований, проведенных зарубежными учеными, можно сделать несколько выводов:

1. Слишком сильная тревога при непредсказуемой угрозе (U-threat) является основной чертой панического расстройства. При этом паническое расстройство характеризуется периодами острого страха, длительной тревоги и беспокойства между приступами паники. Анализ отдельных теоретических моделей панического расстройства показал, что слишком сильная тревога во время неопределенности при панической атаке увеличивает вероятность дополнительных атак. (Lynne Lieberman, Stephanie M Gorka , Stewart A Shankman, K Luan Phan,2016).

2. Специфика развития панических атак связана 
с уровнем аутоагрессии, агрессивно-защитной позицией по отношению к окружающему социальному пространству. (Andrew J. Baillie, Ronald M.Rapee.,2004).

3. Люди с обсессивно-компульсивным и паническим расстройством обладают меньшей когнитивной гибкостью, у них преобладают дисфункциональные метакогнитивные убеждения и отрицательные убеждения относительно эмоциональных схем. (GulayOguz ,Zekiye Celikbas, Sedat Batmaz, Saime Cagli1,Mehmet Zihni Sungur,2019).

4. При нарушении регуляции эмоций панические атаки и стресс переживаются тяжелее. Результаты исследования показывают, что нарушение регуляции эмоций может влиять на то, как люди оценивают симптомы, связанные с паникой (Matthew T. Tull, 2006).

5. Одной из причин панических атак и повышенной агрессии являются родовые травмы и образ жизни матери в период беременности. Например, применение анестетиков при родах приводит к формированию базового чувства недоверия к миру.

Во время утробной жизни депрессия или хроническая тревога у матери могут навредить ребенку. Если мать сидит на диете, то у ребенка может возникнуть рас- стройство пищевого поведения. Если в утробе матери ребенок испытывал голод, то, у него остается страх голода и, он может переедать.

Человек может не осознавать причин паники или агрессии, потому что они появились из-за родовых травм. Очень часто психотерапия не дает нужного эффекта из-за того, что не работает с первичными причинами страхов, фобий и агрессии. (Arthur Janov,2017).

Несмотря на то что проводится множество исследований на тему панических, тревожных расстройств, изучаются различные методики и их эффективность при лечении этих расстройств. До сих пор помощь, которая оказывается больным с паническими расстройствами недостаточно эффективна. Это связано во многом с редким использованием комплексного подхода к лечению, с отсутствием четко определенных критериев использования различных методов лечения. Часто лечение панических атак осуществляется без учета особенностей депрессивных состояний, в структуре тревожных расстройств.

Для успешного лечения панических атак и других тревожных расстройств требуется комплексный подход с изучением причин, факторов риска и прочих особенностей панических расстройств.

\section{ЛИТЕРАТУРА}

1. Andrew J. Baillie, Ronald M. Rapee. Panic attacks as risk markers for mental disorders/Andrew J. Baillie, Ronald M. Rapee. — Текст : электронный // Social Psychiatry and Psychiatric Epidemiology, 06 0ct., 2004. URL: https://link.springer.com/article/10.1007/s00127-005-0892-3 //

2. ArthurJanov. THE ORIGIN S OF AN XIETY, PAN IC AND RAGE ATTACKS/Janov A. — Текст : электронный // Activitas Nervosa Superior volume, Feb.21,2017. URL: https://link.springer.com/article/10.1007/BF03379596//

3. Claire M Kelly 1, Anthony F Jorm, Betty A Kitchener. Development of mental health first aid guidelines for panic attacks: a Delphi study/ Claire M Kelly 1, Anthony F Jorm, Betty A Kitchener. — Текст : электронный // BMC Psychiatry volume 9, Aug. 10, 2009. URL: https://link.springer.com/article/10.1007/BF03379596//

4. Gulay Oguz, Zekiye Celikbas, Sedat Batmaz, Saime Cagli1,Mehmet Zihni Sungur. Comparison Between Obsessive Compulsive Disorder and Panic Disorder on Metacognitive Beliefs, Emotional Schemas, and Cognitive Flexibility/ Gulay Oguz,Zekiye Celikbas,Sedat Batmaz,Saime Cagli1,Mehmet Zihni Sungur. — Tekct : электронный // International Journal of Cognitive Therapy, Jul. 09, 2019. URL: https://link.springer.com/article/10.1007/s41811-019-00047-5

5. Kirsten A. Johnson, Erin C. Berenz, Michael J. Zvolensky. Nonclinical Panic Attack History and Anxiety Sensitivity: Testing the Differential Moderating Role of SelfReport and Behavioral Indices of Distress Tolerance/ Kirsten A. Johnson, Erin C. Berenz, Michael J. Zvolensky. — Текст : электронный // Cognitive Therapy and Research volume, 0ct. 30, 2011. URL: https://link.springer.com/article/10.1007/s10608-011-9410-y/

6. Lynne Lieberman, Stephanie M Gorka , Stewart A Shankman , K Luan Phan . Impact of Panic on Psychophysiological and Neural Reactivity to Unpredictable Threat in Depression and Anxiety/ Lynne Lieberman, Stephanie M Gorka , Stewart A Shankman , K Luan. — Текст : электронный // Clinical Psychological Science 1-12, Oct. 21, 2016. URL: https://pubmed.ncbi.nlm.nih.gov/28138418//

7. MatthewT. Tull. Extending an Anxiety Sensitivity Model of Uncued Panic Attack Frequency and Symptom Severity: The Role of Emotion Dysregulation/ Tull Matthew T. — Текст : электронный // Cognitive Therapy and Research, Jun. 22, 2006. URL: https://link.springer.com/article/10.1007/s10608-006-9036-7 